\title{
A Hot-Cold Cognitive Model of Depression: Integrating the Neuropsychological Approach Into the Cognitive Theory Framework
}

\author{
Elayne Ahern $^{\mathrm{ab}}$, Claudi L. H. Bockting ${ }^{\mathrm{c}}$, Maria Semkovska ${ }^{\mathrm{ab}}$ \\ [a] Department of Psychology, University of Limerick, Castletroy, Limerick, Ireland. [b] Health Research Institute, \\ University of Limerick, Castletroy, Limerick, Ireland. [c] Department of Psychiatry, Amsterdam UMC, University of \\ Amsterdam, Amsterdam, The Netherlands.
}

Clinical Psychology in Europe, 2019, Vol. 1(3), Article e34396, https://doi.org/10.32872/cpe.v1i3.34396

Received: 2019-03-08 • Accepted: 2019-07-08 • Published (VoR): 2019-09-20

Handling Editor: Cornelia Weise, Philipps-University of Marburg, Marburg, Germany

Corresponding Author: Elayne Ahern, Department of Psychology, University of Limerick, Castletroy, Limerick, V94 T9PX, Ireland. E-mail: elayne.ahern@ul.ie

\begin{abstract}
Background: In the 50 years following Beck's cognitive theory, empirical research has consistently supported the role of dysfunctional, 'hot' cognition in the onset and maintenance of major depressive disorder. Compromised 'cold' cognition in attention, memory, and executive control abilities, independent of the affective state, has attracted much clinical interest for its role throughout the course of illness and into remission. We propose integrating cold cognition into Beck's cognitive theory framework to account for the complementary roles of both hot and cold cognition in depression onset and maintenance.

Method: A critical review of cognitive research was conducted to inform an integrated hot-cold cognitive model of depression.

Results: Cold cognitive deficits likely act as a gateway to facilitate the activation and expression of the hot cognitive biases through a weakened ability to attend, retrieve, and critically assess information. Cold deficits become exacerbated by the negative mood state, essentially 'becoming hot', lending to maladaptive emotion regulation through ruminative processes. Depleted cognitive resources contribute to the manifestation of further deficit in problem-solving ability in everyday life, which in itself, may act as a stressor for the onset of recurrent episodes, perpetuating the depressive cycle.

Conclusion: We discuss the interaction between hot and cold cognition within the cognitive theory framework and the potential of complementary hot-cold pathways to elucidate novel means of prevention and treatment for depression.
\end{abstract}




\section{Keywords}

cognition, cognitive behavioural therapy, cognitive control, cognitive dysfunction, cognitive training, depression, emotion regulation

\section{Highlights}

- Dysfunction in hot (emotion-dependent) and cold (emotion-independent) cognition has been demonstrated in depression, but psychological treatment has largely focused on hot cognition only.

- Hot and cold cognition are complementary processes throughout the activation and maintenance of depressive schemas and biases, necessitating the consideration of both hot and cold cognitive aspects to disrupt the depressive cycle.

- The proposed hot-cold cognitive model shows promise to stimulate new research avenues for the prevention and treatment of depression.

Major depressive disorder (MDD) is debilitating, usually chronic in nature, and widely prevalent, affecting approximately 300 million individuals at any given time (World Health Organization [WHO], 2018). As the likelihood of recurrence and relapse remain high (Boland \& Keller, 2009), the need to consider novel treatment strategies and adapt current treatments for MDD is mounting. An examination of the potential mechanisms underlying the central features of MDD may help inform initiatives to optimise treatment outcomes.

Cognitive dysfunction is an important diagnostic feature of MDD. It is best conceptualised as two interacting systems of cold and hot cognition (Roiser \& Sahakian, 2013). Cold cognition refers to information processing that occurs independent of any emotional influence. Such cold cognitive functions are typically assessed using neuropsychological test batteries and include measures of the ability to memorise and retain new information, divide/shift attention between tasks, and make/follow an organised plan in the context of non-affective, neutral stimuli (i.e. no intentional positively or negatively valenced material is provided during the test). Cold cognitive dysfunction has been demonstrated throughout the course of MDD, with broad deficits observed across attention, memory, and executive function measures (Rock, Roiser, Riedel, \& Blackwell, 2014; Snyder, 2013). Meta-analytical evidence show such deficits to be present from the first episode (Ahern \& Semkovska, 2017), while research also suggests a degree of persistence in some cognitive domains beyond the restoration of mood (Semkovska et al., 2019). On the other hand, hot cognition is reactional to the presence of emotional stimuli (e.g. facial expression, affective words, music), or may be a response to feedback that produces an emotional state (e.g. disappointment). For example, individuals with MDD have demonstrated better memory recall for negative stimuli in comparison to healthy controls, sug- 
gesting that MDD has a distinct hot cognitive profile characterised by mood-congruent biases in information processing and memory (Gaddy \& Ingram, 2014). A particular style of perseverative, negative thinking, referred to as rumination (Nolen-Hoeksema, 1991), also largely contributes to the hot cognitive profile characteristic of MDD. This is considered a maladaptive emotion regulation strategy that serves to maintain the negative mood state.

Cold cognitive dysfunction is an area of increased clinical research focus, not only because it can be considered a biomarker for vulnerability (Allott, Fisher, Amminger, Goodall, \& Hetrick, 2016), but also because it has been shown to predict poor treatment response (Groves, Douglas, \& Porter, 2018), and impede functional recovery from MDD (Bortolato et al., 2016). Accumulating literature suggests that the cold cognitive dysfunction associated with MDD drives the negative consequences for everyday life and economic costs, over and above the influence of mood symptoms (Buist-Bouwman et al., 2008). Cold cognitive abilities have also been identified as the single best longitudinal predictor of socio-occupational functioning among young psychiatric outpatients (Lee et al., 2013). Consequently, cold cognition has been increasingly recommended by clinical research as an essential therapeutic target to ensure functional recovery following a depressive episode (e.g. Bortolato et al., 2016). Antidepressant treatment for MDD has demonstrated efficacy on both the affective (mood state) as well as the cold cognitive aspects of depression, most notably among executive functions (e.g. Baune \& Renger, 2014). While improvements have been observed in certain cold cognitive abilities, not all improve at a similar rate; persistent deficits have been noted across attention, verbal memory, response inhibition, decision speed, and information processing in up to $95 \%$ of individual cases (Shilyansky et al., 2016). Although the pro-cognitive effect of antidepressants may not be consistently demonstrated, cold cognition at the very least has been recognised as a potential therapeutic target within the biological approach to MDD treatment. The psychological approach has largely been informed by Beck's cognitive model, thus establishing a focus on hot cognition. Beck conceptualised cognition during MDD as rigid negative schemas and dysfunctional attitudes about the self, the world, and the future. Such problematic schemas and attitudes were theorised to contribute to negative automatic thoughts and biases in attention, information processing, and memory (Beck, 2008). These hot cognitive features interacted, steering the onset and maintenance of MDD. Beck's cognitive model has been central to our understanding and treatment of MDD for the past 50 years. Nevertheless, it has also contributed to an under-emphasis of the role of cold cognitive abilities (Knight, Aboustate, \& Baune, 2018), some of which have been outlined in the DSM diagnostic criteria for MDD, e.g. diminished ability to think or concentrate, and indecisiveness (American Psychiatric Association, 2013). Cognitive behavioural therapy (CBT), which is informed by Beck's cognitive model, is considered the gold standard approach for the psychological treatment of MDD. Interestingly, the effect of psychotherapies such as CBT on cold cognition is not well explored (e.g. 
Porter et al., 2016) as their recognised efficacy is based on the successful treatment of the hot dysfunctional cognitions. Considering patient preference for psychotherapy relative to pharmacological treatment is over threefold (McHugh, Whitton, Peckham, Welge, \& Otto, 2013), novel treatment adjuncts should be explored so that they can guide and ultimately help integrate cognitive remediation strategies into existing psychological treatment.

Contemporary theoretical approaches have brought focus to cognitive control dysfunction during the characteristic depressive biases to account for symptom onset and maintenance (De Raedt \& Koster, 2010; Gotlib \& Joormann, 2010; Joormann \& Vanderlind, 2014). Such approaches propose that MDD is accounted for by an attenuated ability to inhibit the processing of negative material in working memory along with deficits in removing negative material and updating the contents of working memory. Nevertheless, the role of cognitive dysfunction, in both hot and cold forms, has not been formally integrated into the classical cognitive model of MDD proposed by Beck. Typically, hot and cold cognition have been investigated separately within the MDD literature, but we propose integrating hot and cold cognition considering their close alignment and potential complimentary processes as a cognitive mechanism by which negative schemas and cognitive biases contribute to depressive symptoms. The pivotal role that cold cognitive abilities may play in onset, throughout the depressive mood state, and into remission require attention in psychological, theoretical models to account for the dynamic interplay between cognition and emotion. This advancement is necessary to inform treatment strategies that can potentially optimise functional recovery from MDD. Hence, the aim of this paper is to propose a reframing of Beck's cognitive model to integrate a narrative on the role of cold cognition in the development, maintenance, and recovery from MDD. In subsequent sections, we will critically discuss the interaction between hot and cold cognition, followed by a review of the relevance of cold cognition for the cognitive model. Thereafter, we will address the dynamic, interactive processes of hot and cold cognition with reference to each of the cognitive biases in attention, information processing, and memory recall, as outlined by Beck. Finally, we will present our integrated hot-cold cognitive model of depression.

\section{Hot and Cold Cognition}

MDD is often conceptualised within an emotion dysregulation framework; the individual regulates emotion less effectively as a result of a compromised interaction between emotional and cognitive processes, outlining an emotion-cognition link in MDD (Gotlib \& Joormann, 2010). Specifically, cognition has been proposed as the means by which emotion is regulated (Joormann \& Vanderlind, 2014). Cognitive biases affect emotion regulation by drawing attention to emotion-eliciting details or appraisals that are congruent with the negative mood. In turn, deficits in executive functions increase the likelihood of 
using maladaptive regulatory strategies such as rumination, which only serve to intensify and prolong the experience of negative mood (Joormann, 2010; Koster, De Lissnyder, Derakshan, \& De Raedt, 2011). Executive functions, also referred to as cognitive control within the cognitive neurosciences, regulate information processing and behaviour to align with current goals when faced with interference from task-irrelevant stimuli or automatic responses (Friedman \& Miyake, 2017). The processes involved in cognitive control are typically operationalised into shifting (switching between tasks, i.e. cognitive flexibility), inhibition (overriding a prepotent response), and updating (monitoring the contents of working memory; Miyake et al., 2000). An accumulating body of research over the past decade has supported the role of cognitive control dysfunction in MDD for symptom onset and maintenance, but this has largely been investigated in relation to the emotional state. As hot cognition accounts for information processing and reasoning that is influenced by emotional state, weakened cognitive control during MDD is probably best conceptualised as a dysfunctional hot cognition within current theoretical accounts (De Raedt \& Koster, 2010; Joormann, 2010; Joormann \& Vanderlind, 2014). Consistent with this, Gotlib and Joormann (2010) have acknowledged that cognitive control deficits appear to manifest following affective interference and are particularly salient during the processing of negative material, rather than representing a more generalised deficit in cognitive functioning. The complexities of the emotion-cognition link could, nevertheless, benefit from consideration outside of the emotional context by incorporating cold cognition into the explanatory framework. In accordance with this, recent empirical research has suggested that non-affective cognitive control training (Paced Auditory Serial Addition Task, PASAT; Gronwall, 1977) can successfully alleviate residual depressive symptoms among a remitted MDD sample, demonstrating that cold cognitive control abilities may have preventative potential for recurrent MDD episodes (Hoorelbeke \& Koster, 2017). This could result from a disruption to the dynamic interaction between hot and cold cognition following the cognitive training, an interaction which may be necessary for episode onset. Importantly, cold cognitive control deficits are observed during the acute depressive state, with meta-analytical findings from 113 studies providing robust support for deficits in shifting, inhibition, and updating of a moderate effect size among individuals with MDD, relative to healthy controls (Snyder, 2013). Consideration of cold cognition, and particularly the interaction between hot and cold cognitive processes, may be integral to elucidating the mechanisms by which cognition helps regulate emotion. In a critical review of cognitive control theory and research, Grahek, Everaert, Krebs, and Koster (2018) reinforced the importance of considering interactive processes in dysfunctional cognition during depression, with particular acknowledgement of the potential role that hot components (via motivation) may play in cognitive control abilities.

Although hot and cold cognition have been presented as somewhat separate processes, their interaction is central to the maintenance of the depressive cycle. Indeed, per- 
formances on cold cognitive tasks are thought to be partially explained in terms of an altered hot cognition generated by task feedback. As such, Beats, Sahakian, and Levy (1996) demonstrated that negative feedback on a non-affective, neutral task can stimulate a ruminative thinking style on performance or a 'catastrophic response to perceived failure' that, in turn, impairs subsequent performance; intrusion effects generated by negative feedback can slow performance or lead to attention/distraction errors. Furthermore, such feedback can lessen motivation to proceed. In this way, cold cognition can 'become hot', a hypothesis that has been supported by several studies (Murphy, Michael, Robbins, \& Sahakian, 2003; Pizzagalli, Peccoralo, Davidson, \& Cohen, 2006), although such results are not always consistently replicated. As a notable exception to the above hypothesis, Douglas and Porter (2009) showed that individuals with MDD can also improve their performance after perceived failure, albeit to a lesser extent relative to healthy controls. Additionally, Aker, Bø, Harmer, Stiles, and Landrø (2016) demonstrated that remitted MDD individuals did not differ from healthy controls in post-error speed of adjustment on two cold cognition tasks assessing inhibition abilities, despite a slower performance by MDD individuals on one of these tasks. This suggests that the error-feedback provided during task performance did not exacerbate cold deficits by means of hot interference via ruminative processes or depleted motivation. Throughout the literature, deficits in cold cognition are demonstrated even when no feedback on performance is available, suggesting that a negative, hot feedback loop is not necessarily generated during cold cognitive tasks that can explain subsequent performance (Roiser \& Sahakian, 2013).

The distinction between hot and cold cognition is somewhat arbitrary as both mutually influence each other, but it does direct attention to the fact that MDD is associated with compromised function in emotion-dependent (hot) and emotion-independent (cold) cognitive processes. The association between mood and cognitive deficits during MDD has stimulated much critical debate in an attempt to determine whether cognitive deficits (a) are a vulnerability trait that precede and contribute to the onset of a depressive episode, remaining stable throughout into periods of symptomatic remission (b) represent a state by-product of depressive mood, alleviating with mood restoration or (c) develop during the depressive episode and persist as a residual scar (for further detail on trait, state, and scar patterns of cognitive deficit, see Ahern \& Semkovska, 2017). It is likely that each of the above is relevant during the course of MDD from acute phases through to remission and relapse. Although research interest on the role of cold cognition in the course of MDD is relatively recent, it represents an area ripe for further inquiry considering the complimentary processes of cold cognition with the well-recognised hot cognitive processes. A better understanding of the role of these cold cognitive abilities within the cognitive theory framework may elucidate novel means of preventative and treatment strategies. 


\section{Relevance of Cold Cognition for the Cognitive Model}

Beck's cognitive model has outlined the central role of hot cognitive biases to the onset, maintenance, and remission of MDD. Such biases are proposed to result from negative schemas that remain latent until activated by a stressful life event resulting in a change in information processing in a schema-congruent manner (Beck, 2008). However, not all cases of MDD onset are preceded by a stressful life event (Hammen, 2005), suggesting that hot cognitive biases may indeed be a correlate of depressive symptoms rather than an initiating event for onset. Ample cross-sectional research confirms the interaction between seemingly cold cognitive deficits and mood throughout the course of MDD (e.g. Rock et al., 2014; Snyder, 2013), albeit not explicitly measuring the potential explanatory role of hot cognition such as cognitive biases. Nevertheless, it has been suggested that cold deficits precede, and therefore may facilitate, the cascade of hot cognitive biases that occur with MDD onset by compromising the ability to attend, remember, and critically assess information (Knight et al., 2018). Consequently, the individual becomes susceptible to negative information processing. De Raedt and Koster (2010) proposed that weakened cognitive processes, particularly in attention, act as a gateway for negative thoughts and biases. As such, deficits in inhibition contribute to a repetitive cycle of negative thoughts, while deficits in shifting exacerbate the ability to move away from or disengage attention from these thoughts, resulting in a maintenance of depressive mood. Deficient cognitive control of negative information is considered central to the maintenance of hot cognitions and depressive mood, but this does not explain why individuals with MDD often continue to demonstrate compromised functioning when mood has alleviated. Cold cognitive deficits may be an important consideration to explain the continued compromised functioning during remission, but also may help elucidate a better understanding of how the cognitive and affective components of MDD interact to initiate and sustain depressive symptoms. The conceptual framework proposed by De Raedt and Koster (2010) integrated biological and cognitive factors to outline the potential working mechanisms involved in vulnerability for recurrent MDD episodes. Hot attentional deficit, where attention is diverted to and maintained on negative material, was a central component of the framework to account for sustained negative affect through interaction with the activated schemas. Although De Raedt and Koster (2010) acknowledged cold deficits in attention, the hot attentional component was considered of particular interest due to its depression-specificity and that it could be explained from a biological perspective (i.e. as a consequence of reduced cognitive control exerted by the dorsolateral prefrontal cortex [due to deficient signals from the anterior cingulate cortex] on the emotional processing conducted by the amygdala). Thus, the framework proposed by De Raedt and Koster (2010) mainly accounted for the role of hot attentional deficits in the vulnerability for recurrence. 
It is plausible that cold deficits represent the vulnerability that interacts with the schema to generate the characteristic hot cognitive bias during MDD. Weak inhibitory and shifting processes have been shown to be associated with the development of depressive symptoms up to 7.5 years later among youth, while controlling for other key predictors such as gender and IQ (Kertz, Belden, Tillman, \& Luby, 2016). These findings suggest that cold cognitive control deficits likely precede and represent a vulnerability factor for onset. Further corroborating this, deficits in cognitive control measures such as working memory, shifting, and inhibition have been noted at initial MDD onset, with broader deficits demonstrated across processing speed, attention (visual and auditory), learning and memory (visual and verbal), reasoning, verbal fluency, motor skills, and intelligence, with the magnitude of deficit varying from small to large across these cognitive domains (Ahern \& Semkovska, 2017). As these deficits cannot be accounted for by the additive effect of scarring from recurrent episodes, it is reasonable to assume that cold cognitive dysfunction can precede onset. Furthermore, executive function deficits have even shown stable, trait-like qualities from the first depressive episode (Ahern \& Semkovska, 2017). Snyder and Hankin (2016) suggested that cold executive control deficits are linked to internalising psychopathologies through stress generation and subsequent rumination. Cold deficits contribute to self-generated stressors (e.g. difficulties at work due to poor planning of time/approach to workload), and while stressors are widely acknowledged as risk factors for the development of psychopathology (e.g. Grant et al., 2014), this initiating mechanism represents a novel means to better understand the MDD cycle. Perhaps it could even be postulated that the cold cognitive deficits themselves represent a self-generated stressor when the individual is made self-aware of deficient functioning in day-today tasks.

Beck's cognitive model proposed that dysfunctional schemas remained dormant until activated by a stressor (Beck, 2008). Along these lines, cold cognitive deficits across a broad range of cognitive domains may serve as internal, self-generated stressors that offset the cascade of hot dysfunctional cognitions and biases during MDD. This assumes the affective interference hypothesis in that affective information becomes salient and impedes on the subsequent processing of information (Siegle, Ingram, \& Matt, 2002). In this way, seemingly cold cognitive deficits 'become hot' (e.g. in response to feedback) as the MDD cycle is initiated. It has been suggested that cognitive deficits are superimposed as a result of a 'catastrophic response to perceived failure' when the activated negative schema biases processing of the feedback which, in turn, impedes on subsequent performance (Elliott et al., 1996). Beats et al. (1996) demonstrated using the Tower of London task that MDD individuals solved as many problems in the minimum specified moves as did controls, but once a mistake was made, subsequent performance deteriorated rapidly. Further to this, Elliott, Sahakian, Herrod, Robbins, and Paykel (1997) showed that MDD individuals underwent more errors on a series of CANTAB tasks (Delayed Matching to Sample and 1-touch Tower of London) in response to feedback, relative to controls and 
other clinical groups (e.g. schizophrenia). Findings by Beats et al. (1996) and Elliott et al. (1997) suggest that cognitive deficit on neutral, non-affective tasks are largely just a manifestation of hot cognition, likely due to a ruminative response style centred around the negative feedback.

Once initiated, the hot dysfunctional schemas and cognitive biases place demands on cognitive resources as they commence a sequence of automatic, mood-congruent processing, relying on executive function, memory, and attention abilities to maintain the depressive mood state. The resource allocation hypothesis postulates that the depletion of available cognitive resources has a detrimental effect on day-to-day cognitive functioning as limited resources are available to direct to other non-affective, cold cognitive functions (Ellis \& Ashbrook, 1988), overall exacerbating the cognitive profile of MDD. Similarly, as limited cognitive resources are available to engage in effortful, controlled processing to override the automatic biases, maladaptive regulation strategies such as rumination ensue as subsequent hot cognition. Consequently, hot and cold dysfunction initiate a mutually reinforcing cycle. We propose that the dynamic interaction between hot and cold cognitive processes is central to MDD and necessitates consideration of both to stimulate theoretical advances and integrated research efforts.

In the above section, we have outlined the basic tenet of our integrated hot-cold cognitive model of MDD. In the following sections, we discuss the interaction between hot and cold in the context of the cognitive model with particular reference to the characteristic biases in attention, information processing, and memory recall during MDD (Gotlib \& Joormann, 2010). These hot-cold interactions are illustrated through Figure 1, 2, and 3 , respectively. Although it is acknowledged that the cognitive biases likely influence each other and interact to exert influence on other factors (Sanchez, Duque, Romero, \& Vazquez, 2017), we will discuss each of the cognitive biases separately. This is consistent with the approach of Disner, Beevers, Haigh, and Beck (2011) in their work on the neural mechanisms underpinning Beck's cognitive model. The authors demonstrated that although cognitive biases are characterised by increased bottom-up emotional processing and attenuated top-down cognitive control, the underlying mechanisms of each cognitive bias somewhat differ. Thus, modelling each cognitive bias separately will allow us to best account for the interactive role of hot and cold cognitions and essentially advance our understanding of the maintenance and treatment of MDD.

\section{Biased Attention for Negative Material}

A compromised ability to direct attention to relevant information is central to the cognitive model of MDD. This is driven by an apparent attention bias towards negative stimuli, although its existence is not robustly supported throughout the literature (Mathews \& MacLeod, 2005). Williams, Watts, MacLeod, and Mathews (1988) even suggested that MDD is characterised by biases in elaboration and recall but not an attention bias, which is more so characteristic of anxiety disorders. Attention biases in MDD have been exam- 
ined in the early and later stages of attentional processing in an attempt to better determine whether, and under what conditions, attentional dysfunction is observed. The initial orienting of attention has been investigated using subliminal or rapid presentation of affective material. For example, using an emotional Stroop task in which valenced-words were presented against a background colour for approximately $1 \mathrm{~ms}$ and followed by a mask, Mogg, Bradley, Williams, and Mathews (1993) showed that preattentive bias for negative information occurred in the anxiety group only, while the MDD group were comparable to controls. The absence of attention bias in the early stages of processing has been replicated throughout the literature (for review, see Mogg \& Bradley, 2005). Nevertheless, research on attention bias at later stages of conscious processing provides more robust evidence for the presence of a mood-congruent bias (e.g. Gotlib,

Krasnoperova, Yue, \& Joormann, 2004), and once attention is directed to the negative material, MDD individuals typically spend longer periods of time engaged with it (e.g. Kellough, Beevers, Ellis, \& Wells, 2008). This suggests that MDD is not characterised by an automatic attention bias, as proposed by the cognitive model, but instead is associated with difficulty disengaging from negative material once attended to. As a result, a positive feedback loop is initiated in which depressive symptoms are maintained by the interaction between hot attentional control deficits and rumination. Impaired disengagement from negative, self-relevant thoughts maintains an inward focus that strengthens ruminative processes, overall serving to worsen the depressive mood (Koster et al., 2011). Findings by Yaroslavsky, Allard, and Sanchez-Lopez (2019) supported the mediational role of rumination between hot attentional disengagement deficits (slow disengagement from sad faces, fast disengagement from happy faces) and depressive symptoms. This suggests that hot cognitive deficits manifest in subsequent forms of hot cognition, namely rumination, to maintain depressive symptoms. In addition, experimental research using attention bias modification strategies has corroborated the mediational role of rumination in the link between attention bias and depressive symptoms (Yang, Ding, Dai, Peng, \& Zhang, 2015). Attention bias modification strategies typically involve training the individual to direct attention away from a negative stimulus towards a neutral stimulus. This is often achieved using a dot-probe paradigm where the target probe is located more frequently behind the neutral stimulus when presented simultaneously with a negative stimulus; after repetition, this training gradually helps to facilitate a more positive attention bias. Following eight sessions of attention bias modification (90\% of targets appeared in the neutral word position, relative to the sad word position) over a 2-week period, Yang et al. (2015) demonstrated that depressive symptoms significantly improved and were maintained at 3-month follow-up; no improvements were shown for the control attention training ( $50 \%$ of targets appeared in the neutral/sad word position) or assessment-only conditions. In addition, more participants remained asymptomatic at 7month follow-up subsequent to attention bias modification, relative to the comparison conditions. Findings showed that this effect was fully mediated by rumination and, there- 
fore, change in attention bias could not directly account for improved depressive symptoms. Hence, it is suggested that deficient hot cognitive control abilities consequently manifest as a ruminative response style as a result of impaired disengagement from negative material. This process overall sustains the negative affect and associated attention bias.

Nevertheless, findings have not unequivocally converged to demonstrate that targeting hot cognition alone can account for depressive symptom improvement. Considering control attention training has often shown similar efficacy to attention bias modification strategies, cold cognitive abilities may also play a potential role in symptom improvement. Beevers, Clasen, Enock, and Schnyer (2015) found that symptom improvement was not significantly different for control attention training (50\% of targets appeared in neu$\mathrm{tral} /$ negative location) relative to a homework supplemented, 4-week attention bias modification training consisting of eight sessions (80\% of targets appeared in the neutral location, relative to the negative location); across the training groups, an overall $40 \%$ symptom improvement was observed. Such findings suggest different mechanisms of symptom improvement via hot and cold processes, although this warrants further investigation to better understand the driving mechanism. In the attention bias modification condition, symptom change may be somewhat accounted for by change in attention bias, yet for the control attention training, benefits may have been a result of engaging in an activity that promoted focused and sustained attention. This allows us to postulate that cold attention abilities are involved in symptom improvement but also that their dysfunction may represent the potential mechanism by which hot attention biases materialise following affective interference. Additionally, Jonassen et al. (2019) showed that attention bias modification training ( $87 \%$ of targets appeared in the positive location, relative to the negative location), when completed twice daily over a 2-week period, did not significantly differ from control attention training (50\% of targets appeared in the positive/negative location), with both contributing to significant self-reported depressive symptom improvement. Notably, significant, albeit smaller, improvements on clinician-rated depressive symptoms were found for the attention bias modification group only, with such symptom improvement closely associated with a more positive attention bias. Overall, changes in attention bias did not significantly differ between the training groups, suggesting that attention bias modification may exert its effect by means of another mechanism, potentially by training general attention ability. Typically, the only difference between attention bias modification training and the control training condition is the frequency of target presentation in the negative location. Consequently, session duration or frequency of training cannot account for the lack of differential findings between the active and control intervention conditions, although these factors may become relevant for comparisons between experimental studies. Inclusion of an assessment-only comparison will help to elucidate whether training attention ability, irrespective of an attention bias focus, is the underlying driving mechanism of symptom improvement. 


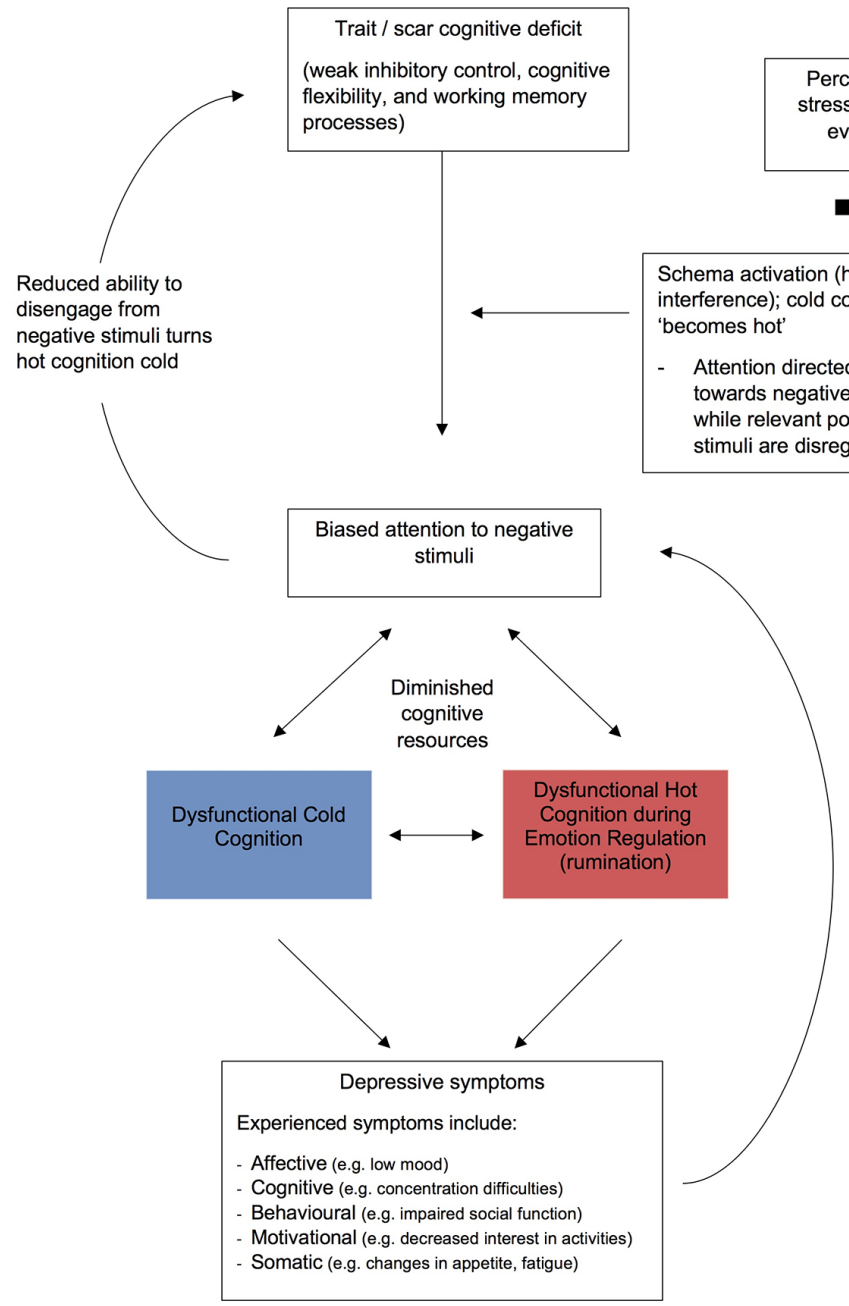

Figure 1. Model of the interaction between cold cognitive deficits and the hot cognitive attention bias during depression.

Note. When triggered by a perceived stressful life event, weaknesses in cognitive flexibility and inhibitory processes interact with the schema (negative core belief, e.g. 'I am useless') leading to a failure in disengaging attention from negative stimuli, i.e. attention bias. Subsequent hot ruminative processes magnify the accessibility of negative material and represent a means by which the bias affects depressive symptoms. There is a consequent reduction in available cognitive resources while hot cognition is activated. This contributes to broader cognitive deficits, including a general attention deficit, which likely lends itself to the expression of the attention bias for negative stimuli. Such deficits impact day-to-day functioning, potentially triggering subsequent hot cognition. The interaction between hot and cold pathways serves to perpetuate the activated schema and attention bias, overall worsening depressive symptoms. Components of the model are informed by Beck's cognitive model of depression. 
In summary, biased attention is understood to be central to the maintenance of MDD. Impaired cognitive control reduces the ability to disengage from negative information, thus contributing to exacerbated hot cognitive processes, such as rumination, which reinforce the attention/disengagement bias to overall maintain depressive symptoms. Cold cognitive deficits appear to equally be relevant as general attention training has been shown to improve depressive symptoms, independent of change in attention bias. This suggests differentiated, but likely complimentary hot and cold cognitive processes.

\section{Biased Information Processing in Preference of Negative Material}

According to the cognitive model, MDD is associated with a pattern of interpretation in which stimuli tend to be interpreted negatively (Gotlib \& Joormann, 2010). Resultantly, a positive blockade is experienced as reduced processing abilities contribute to poorer processing of positive stimuli alongside a decreased salience of positive stimuli (Nutt et al., 2007). The MDD interpretation bias is considered central to symptom maintenance. Literature has supported that individuals with MDD tend to employ negative interpretations of ambiguous information, relative to healthy controls, using a variety of methods (for review, see Everaert, Podina, \& Koster, 2017). For example, Butler and Mathews (1983) showed that when asked to interpret an ambiguous sentence from a given list of options, MDD individuals selected the negative option more frequently than controls. However, such findings in the literature have been critiqued for over-reliance on self-report measures, which likely better capture a response bias as opposed to a negative interpretation bias. A response bias could facilitate a pattern of responding that presents as an interpretation bias as individuals with MDD tend to choose negatively-valenced responses when presented as an option, irrespective of their own interpretation of the scenario. In support of this, Lawson and MacLeod (1999) demonstrated that when response time was used as an indicator of interpretation bias, dysphoric individuals did not differ to controls in terms of their reaction time to target negative words following an ambiguous sentence. If an interpretation bias was evident, it could be expected that dysphoric individuals would show faster reaction times to negative words than neutral words as the negative processing bias would prime a negative interpretation. These findings have been replicated (e.g. Bisson \& Sears, 2007), albeit not consistently throughout the literature (e.g. Hindash \& Amir, 2012). Lack of equivocal support throughout the literature could be explained by the severity of depressive symptoms; negative interpretation biases are possibly more pronounced for those with more severe symptoms as a result of exacerbated hot cognition. Consistent with this, Lawson, MacLeod, and Hammond (2002) showed that the magnitude of eye-blink reflex to negative stimuli was larger for those with more severe depressive symptoms, relative to those with less severe symptoms, indicative of greater negative processing.

It is understood that MDD is not driven by the depressive mood nor the depressive thoughts per se, but rather the way in which the individual processes their depressive 
mood. During MDD, this is usually maladaptive processing that involves rumination or repetitive thinking around the causes and consequences of one's mood (NolenHoeksema, 1991). Wisco, Gilbert, and Marroquín (2014) demonstrated that the association between interpretation biases and depressive symptoms was conferred through rumination of negative thoughts as well as dampening of positive thoughts, although the effects through rumination were stronger, comparatively. In addition, reverse mediational analyses provided further evidence of the directionality of effects as the indirect effect of rumination on depressive symptoms through interpretation bias was not significant. As these findings are grounded in correlational research, it is not possible to draw causal conclusions. Nevertheless, such findings suggest that the hot cognitive interpretation bias prompts the hot regulatory process of rumination to promote the maintenance of depressive symptoms. Consideration of longitudinal research by Hirsch et al. (2018), examining the effect of multi-session cognitive bias modification strategies to target interpretation biases, further corroborates this mechanism. This training involved participants listening to scenarios that were emotionally ambiguous but were resolved positively $(76 \%$ of trials), negatively (12\%), or remained unresolved (12\%). In the control, all ambiguous scenarios remained unresolved. At 1-month follow-up, findings suggested that change in interpretation bias following cognitive bias modification partially mediated the interventional effects on rumination but also on depressive symptoms among patients with MDD. This supports the idea that the mechanism by which negative interpretation biases impact depressive symptoms is through further hot ruminative processes. However, active control conditions have previously reduced negative interpretation biases when no training effects were expected (Blackwell et al., 2015; Williams et al., 2015). For example, Blackwell et al. (2015) did not show superior effects on depressive symptoms following multi-session cognitive bias modification, relative to the attention control condition which resolved ambiguous information either positively (50\% of trials) or negatively (50\%). By drawing attention to the fact that outcomes can be resolved positively, and therefore, by training general attention ability, albeit inadvertently, the attention control training still showed benefits for depressive symptoms. Although further research is required to confirm this, it could be argued that the interpretation bias is a manifestation of cold attention deficit that has been 'made hot' by the activated negative schema.

One aspect of biased processing that has drawn interest throughout the cold cognitive deficit literature is feedback sensitivity. As mentioned, seemingly cold cognitive deficits may in fact be hot as a result of a 'catastrophic response to perceived failure' (Elliott et al., 1996), or in other words, affective interference from an activated, negative schema when processing feedback. Individuals with MDD can perform just as well as healthy controls on several measures of cold cognitive abilities, but once feedback on an error is received, performance thereafter is likely reduced (e.g. Beats et al., 1996). Cold cognitive deficits may manifest owing to a repetitive, ruminative response on the feedback that serves to confirm an underlying core belief (e.g. 'I am useless' or 'I am a failure'), thus 
interfering with performance on the task at hand. Elliott et al. (1997) supported this by demonstrating that when MDD individuals were re-assessed upon remission, general cognitive performance on the 1-Touch Tower of London CANTAB task was comparable to healthy controls, but an abnormal response to negative feedback persisted. Thus, the response could not merely be due to an overall higher failure rate. This suggests that hot cognition exerts influence on cold cognitive ability and contributes to deficient performance. However, it is important to note that this finding was exclusive to the 1-touch Tower of London measure in a sample of $28 \mathrm{MDD}$ patients and was not replicated in the Delayed Matching to Sample Test. In a direct comparison between a feedback condition and a no feedback (paired associates) condition, Mörkl, Blesl, Jahanshahi, Painold, and Holl (2016) demonstrated that performance in probabilistic classification learning was impaired during the feedback condition but not for the no feedback condition, relative to controls. Feedback-learning involved receiving a 'thumbs up' or 'thumbs down' in response to the classification of each card to a certain type of weather (rainy/fine). This corroborates that hot information processing biases can manifest as cold cognitive deficits in various domains of learning and attention during seemingly neutral cognitive tasks. Whether this deficit is initiated as an epiphenomenon of the processing bias/feedback sensitivity or is merely exacerbated during the acute depressive state (i.e. 'becomes hot') still warrants further research considering the low-effortful task employed by Mörkl et al. (2016). The consideration of task demands is important when assessing the extent of cognitive control deficits in MDD. Indeed, when tasks are considered more effortful and require more complex processing, the inefficient allocation of cognitive resources to the task at hand means that deficits in cold cognitive functioning become more pronounced (Hammar, Lund, \& Hugdahl, 2003; Hartlage, Alloy, Vázquez, \& Dykman, 1993). Deficits in various cognitive measures including attention, learning and memory, and executive function have been documented in MDD without feedback and therefore cannot merely be a manifestation of feedback sensitivity (e.g. Rock et al., 2014). Nevertheless, a direct comparison of MDD cognitive performance in the presence or absence of feedback would better help to elucidate whether the hot and cold elements involved in the activation and maintenance of the processing bias are mutually reinforcing (i.e. underlying cold deficit is exacerbated by hot ruminative processes following feedback, in turn manifesting as further cold cognitive deficit). Thus, a differential magnitude of deficit may be observed, contingent on the catastrophic response to feedback.

Interestingly, literature has not consistently supported the explanation of a catastrophic response, but rather it is proposed that error post-feedback represents a failure to internalise the feedback through learning and, as a result, performance is not adjusted. The findings of Steele, Kumar, and Ebmeier (2007) align with this interpretation as MDD individuals showed a blunted response following error-feedback in a gambling task suggesting that they did not learn from their past performance. Typically, a 'post-error slowing' is demonstrated where the individual considers the feedback to avoid a repetition of 
error and to adjust performance accordingly. This process manifests as an increase in reaction time. Nevertheless, MDD individuals did not show increased reaction time posterror, suggesting that not even a ruminative response style was employed to process the feedback. Thus, a basic deficit in cold cognitive ability likely accounted for poor performance, not hot ruminative processes. Further to this, using a non-affective flanker task with switch or no-switch blocks (no performance feedback offered), Schroder, Moran, Infantolino, and Moser (2013) demonstrated that anhedonic depressive symptoms were not associated with post-error slowing but were associated with poorer post-error accuracy. As the association between anhedonic depressive symptoms and post-error accuracy was only shown in the more cognitively-demanding switch block condition, the availability of cognitive resources appears to be an important factor for cognitive control ability. These findings allow for the consideration that deficits in attention and the ability to update the contents of working memory during an effortful task account for subsequent poor performance as post-error slowing and deficient post-error accuracy were observed independent of feedback. Although the explanatory potential of deficient motivation in anhedonic depression cannot be overlooked to account for these findings (but see Grahek et al., 2018), the premise that cold cognitive deficits are not merely a manifestation of biased processing is of empirical and theoretical interest.

In summary, the processing bias proposed by the cognitive model appears to be largely maintained by the interaction between hot and cold cognitions. For the most part, ruminative processes carry the effect of this bias on depressive symptoms by stimulating accessible, negative, self-relevant information to reinforce a negative interpretation. Arguably, cold cognitive deficit, in seemingly non-affective contexts, may be a manifestation of hot cognition through a catastrophic response to feedback, and subsequent rumination on this feedback. Nevertheless, cold deficits have reliably be shown in the absence of feedback and symptom improvement has been achieved following control, non-affective attention training, suggesting that there is a complex interaction that likely incorporates both hot and cold cognition as mutually reinforcing, overall serving to maintain depressive symptoms. 


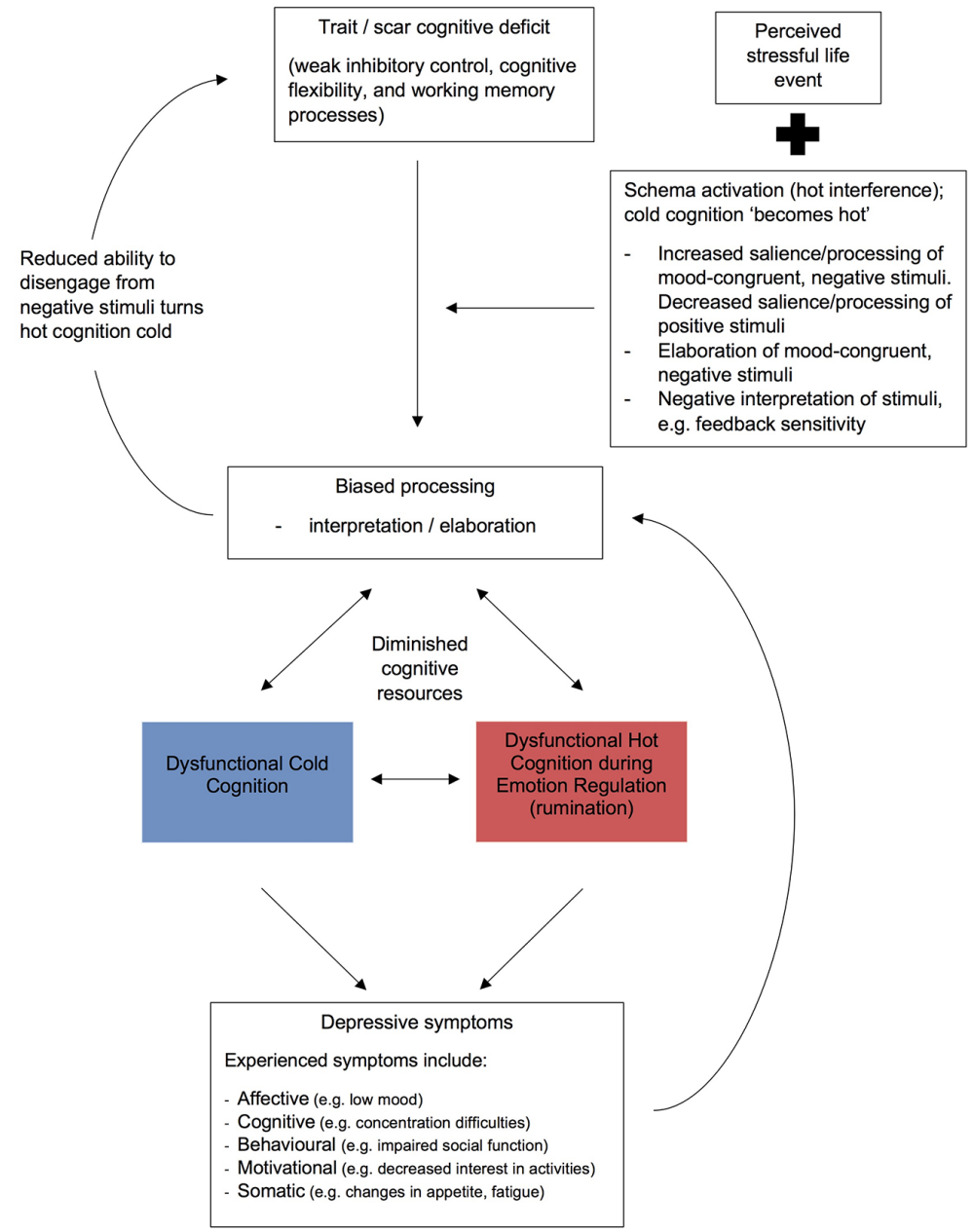

Figure 2. Model of the interaction between cold cognitive deficits and the hot cognitive processing bias during depression.

Note. When triggered by a perceived stressful life event, weaknesses in cognitive flexibility, working memory, and inhibitory processes interact with the depressive schema resulting in a bias towards processing information negatively, congruent with the activated schema. There is a consequent reduction in available cognitive resources when the schema and associated bias is activated. As a result, broader cognitive deficits are observed during the acute depressive state. Specifically, the inability to update the contents of working memory with a shift to positive information (cognitive flexibility) and expel irrelevant, negative information from working memory (inhibition) contribute to rumination, i.e. repetitive, negative thinking style, which further serves to maintain the processing bias and worsen depressive symptoms. Yet, ruminative processes do not always account for cold cognitive deficits in post-error adjustment, suggesting some degree of independence between the hot and cold pathways for biased information processing. Components of the model are informed by Beck's cognitive model of depression. 


\section{Biased Memory for Negative Material}

Biased memory recall during MDD is perhaps one of the most consistent and well-supported cognitive biases (Gotlib \& Joormann, 2010). This is best understood as an instance of memory in which mood-congruent information (i.e. negative information consistent with negative mood state) is better encoded and recalled than mood-incongruent information (Matt, Vázquez, \& Campbell, 1992). Due to the negative mood state that is characteristic of MDD, symptomatic individuals tend to recall negative information more readily as prompted by the accessibility of active, negative schemas, thus serving to maintain the negative mood. This negative recall is likely facilitated by biases at encoding as a result of preferential attention to negative experiences as well as the maladaptive interpretation of ambiguous personal experiences to resolve as negative encounters (Dalgleish \& Werner-Seidler, 2014). Of note, a recall bias in non-affective, cold cognitive tasks has even been observed during MDD, presenting as a serial position effect in list-learning that is characterised by impoverished recall of items from the middle region, relative to healthy controls (Foldi, Brickman, Schaefer, \& Knutelska, 2003). It is postulated that the active, negative schema (e.g. 'I am a failure') interferes with subsequent learning and recall by means of affective interference, drawing focus to task irrelevant information (e.g. 'I will never finish this list'). Consequently, recall has been enhanced for mood-congruent negative information and compromised for incongruent, positive or neutral information, as demonstrated across both explicit (for review, see Matt et al., 1992) and implicit (for review, see Gaddy \& Ingram, 2014) memory tasks. A meta-analysis (Matt et al., 1992) on recall performance among MDD patients demonstrated, on average, that individuals recall $10 \%$ more negative information than positive information on explicit memory recall tasks. A type of explicit memory known as autobiographical memory (AM) is characterised by a particular recall bias during MDD which takes the form of an overgeneralisation, or a lack of specifics in the content recalled, usually in response to negative cues (e.g. Dalgleish \& Werner-Seidler, 2014; Semkovska, Noone, Carton, \& McLoughlin, 2012). Research has demonstrated that overgeneral AM is likely state-dependent, with improvement in specificity shown upon remission (e.g. Semkovska et al., 2012), however, overgeneral AM has also received support as a trait vulnerability for subsequent depressive symptoms, particularly in interaction with stressful life events (e.g. Gibbs \& Rude, 2004).

Overgeneral AM represents a hot cognitive profile that is specific to MDD. This hot cognition has been relatively well-explored in the literature in relation to ruminative processes, which tend to centre around the general themes made accessible by schema activation at recall (e.g. incidences of failure). Rumination then consolidates these themes, which further contribute to overgeneral AM recall (Watkins \& Teasdale, 2001). On the other hand, the seminal work by Raes, Hermans, Williams, Geypen, and Eelen (2006) demonstrated that overgeneral AM can causally influence rumination, following experimental manipulation to prime either a specific or overgeneral recall style. Findings showed that among high-ruminators and subsequent to an overgeneral (vs. specific) re- 
call style, sentences tended to be unscrambled into sentences with a ruminative meaning. Among non- or low-ruminators, this effect was not shown. Thus, it is better to consider the recall bias-rumination link as mutually reinforcing, in that hot cognition stimulates further hot cognition. As such, it could be conceptualised that the effect of memory recall bias on depressive symptoms is carried by a ruminative thinking style. In corroboration of this, Liu et al. (2017) demonstrated that ruminative responses, particularly the maladaptive brooding subtype, partially mediated the relationship between overgeneral AM and depressive symptoms. Albeit, the cross-sectional design cannot infer causality so it is possible that rumination is merely a covariate as opposed to the mediator of effect. The reverse mediational models conducted by the authors, however, did not support overgeneral AM as a potential mediator of the rumination-depressive symptom link. Likely these two hot cognitions are closely intertwined, making it difficult to discern the effect of one on another. Consideration of the role of executive cognitive abilities may better help elucidate the means by which this reciprocal interaction arises.

Ruminative processes that occur during the recall bias use up cognitive resources that otherwise would have been directed towards accessing the specifics of AM (Van Vreeswijk \& de Wilde, 2004). To demonstrate this, Cheung, Sin, Lam, and Lee (2018) assessed the specificity of AM in MDD patients following negative and positive rumination induction. Results showed that MDD patients, relative to controls, produced fewer specific AMs following negative rumination compared to positive rumination. The authors suggested that an inhibitory deficit in MDD compromised the ability to filter out taskirrelevant negative information from working memory, thus impairing the ability to recall specific memories, stimulating a negative ruminative process. Although general inhibitory ability was not assessed by the authors, it could be the case that an underlying cold inhibitory deficit became hot in the presence of affective content. Building on this, Hitchcock, Golden, Werner-Seidler, Kuyken, and Dalgleish (2018) examined the central role of executive control in negative AM recall following positive and negative contextual cues among MDD patients. Results showed that following positive contextual cues, those with more severe depressive symptoms rated their negative memories more positively, relative to less severely depressed patients. As such, this suggests that the executive control deficits are somewhat state-dependent as differential effects were noted based on the magnitude of depressive symptoms. This can be explained by fewer executive resources available in those more severely depressed to override the priming generated by the positive contextual cue. In a second study by the authors using a community sample, poorer performance on a non-affective working memory task was associated with less negative ratings of AMs recalled and with the recall of a greater number of overtly positive AMs. Thus, compromised cold executive control appears to be the driving mechanism of this effect and not a negative recall memory bias, which Beck's cognitive model would hypothesise to occur independent of context. The influence of contextual priming, as shown in these findings, has important implications for mood maintenance. For example, 
if intrinsic contextual cues (i.e. negative thoughts, rumination) override one's ability to derive benefit from the recall of positive or neutral memories necessary for cognitive restructuring, a core aspect of CBT, then outcomes may be less favourable. In this regard, cold executive deficits may play a central role in the maintenance of depressive mood state, over and above those accounted for by hot cognitions.

Overgeneral AM has also been conceptualised as a facet of a wider difficulty in memory processes such as the ability to make correct attributions about the origin of information, known as source memory. Raes, Hermans, Williams, Demyttenaere, et al., 2006) demonstrated that among 26 acute MDD patients, reduced AM episodic-specificity was associated with poorer source memory and working memory, but not verbal fluency or verbal learning and delayed recall. These associations were shown independent of current depressive symptoms. Semkovska et al. (2012) elaborated on these findings among a sample of $30 \mathrm{MDD}$ patients with severe depression, corroborating that reduced AM episodic-specificity was associated with poorer working memory, but that verbal or visual learning and delayed recall were specifically associated with AM semantic-specificity for personal information (e.g. details regarding a family member). Among a remitted MDD sample of 122 individuals, the findings of Spinhoven et al. (2006) strengthen the argument that aspects of reduced AM specificity likely represent a facet of a more general memory deficit independent of the hot affective state, as AM specificity was shown to be associated with immediate and delayed memory recall, even when controlling for residual depressive symptoms. Yet, at 24-month follow-up, neither AM specificity nor other cognitive measures were predictive of relapse or recurrence. This brings to question whether it is the interaction between hot and cold components that may be necessary to trigger subsequent MDD events. Similarly, Gibbs and Rude (2004) suggested that overgeneral AM, when in conjunction with stressful life events, predicted the development of subsequent depressive symptoms 4-6 weeks later in a non-clinical sample. Thus, it could be the case that cold deficits condition, or likewise transfer, the effects of the hot memory recall bias.

The recall of specific memories is a core component of everyday functioning and has relevance for day-to-day tasks that rely on problem-solving, the generation of future plans, and social interactions that are built upon shared experiences (Dalgleish \& Werner-Seidler, 2014). Consequently, cold cognitive abilities are compromised without the script of past AMs to direct and guide behaviour, contributing to subsequent negative experiences in the everyday functioning of the individual. Raes et al. (2005) showed that reduced AM specificity fully mediated the association between rumination and social problem-solving (e.g. handing a situation at work), while partial correlations demonstrated that this association could not be better accounted for by depressive symptoms. Corroborating this, Hitchcock et al. (2016) documented that an AM intervention (MemFlex) showed far-transfer of effects by significantly improving problem-solving ability. Although the authors acknowledged that problem-solving was an intermediate outcome 
that likely conferred the effect of overgeneral AM on depressive symptoms, they did not formally test the mediational process. In addition, planning ability has been observed as a cold cognitive deficit following the induction of a generic retrieval style (Williams et al., 1996). In a recent meta-analysis by Hallford, Austin, Takano, and Raes (2018), psychiatric diagnosis was associated with large deficits in episodic future thinking specificity. Moderation by cue valence (positive, negative, neutral) was non-significant, suggesting that the deficit is more general and not wholly contingent on valence, as would be suggested by Beck's cognitive model. It is important to note, however, that moderation by valence was across all studies $(k=19)$, so it is possible that $\operatorname{MDD}(k=7)$ shows differential effects to other psychopathologies such as bipolar disorder or schizophrenia. A subsequent meta-analysis conducted solely including depressive samples resulted in 37 studies and suggested that depressive symptoms contributed to reduced specificity for future events, more so for positive future thinking relative to negative or neutral future thinking (Gamble, Moreau, Tippett, \& Addis, 2018). Considering the ability to detail future scenarios is necessary to plan and pursue goals, it can be argued that the hot cognitive profile of MDD manifests as a broader spectrum of cold cognitive deficits including planning and problem-solving abilities. Such deficits are likely responsible for the negative experiences of everyday functioning and may serve as a source of reference to confirm and perpetuate the activation of negative schemas (e.g. 'I am a failure'), overall contributing to a maintenance of the MDD cycle.

In summary, the interaction between hot and cold cognitions in the memory recall bias is difficult to disentangle but lends support to the idea that both are mutually reinforcing for the activated bias as well as associated depressive symptoms. Rumination has been identified as the primary hot cognition that carries the effect of the negative recall bias by stimulating negative thoughts around the negative memory that was generated. A cold deficit in non-affective memory potentially facilitates the expression of the aforementioned recall bias, but likely this is determined by the strength of executive control, characterised by the individual's ability to flexibly disengage from mood-congruent negative information, inhibit further irrelevant negative information, and subsequently update the contents of working memory. Without the specifics in AM to provide a script to guide behaviour, a broader range of cold deficit is experienced, which impacts on efficient day-to-day functioning. Collectively, this may serve as self-referent negative information to maintain the activation of the relevant schema and recall bias. 


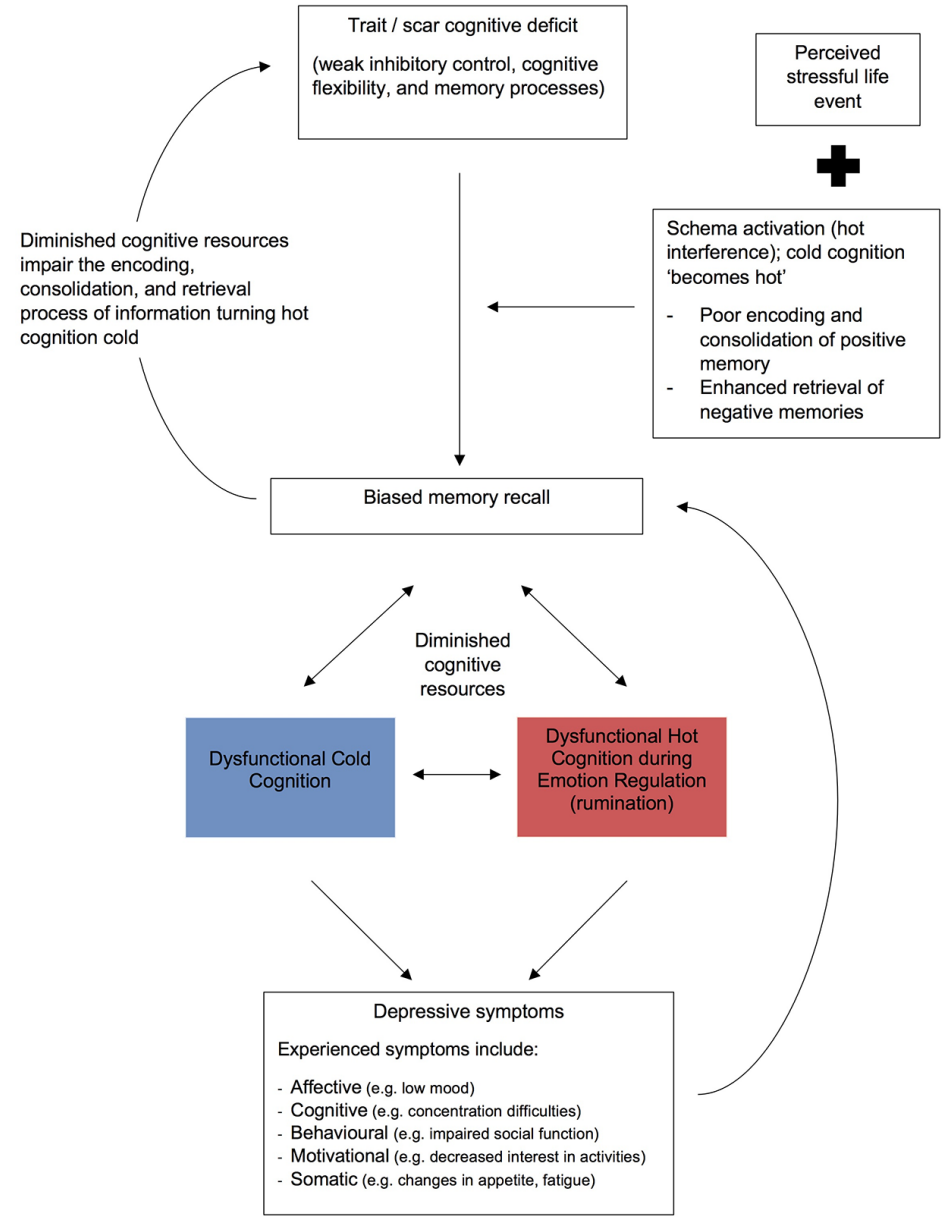

Figure 3. Model of the interaction between cold cognitive deficits and the hot cognitive memory bias during depression.

Note. When triggered by a perceived stressful life event, weaknesses in cognitive flexibility, memory, and inhibitory processes interact with the depressive schema resulting in a memory bias characterised by poor encoding of positive memories, over-retrieval of negative memories, and lack of specificity for autobiographical memories. There is a consequent reduction in available cognitive resources when the schema and associated bias is activated. As a result, deficits in hot and cold executive control perpetuate the activation of the memory bias due to a compromised ability to inhibit, shift away from, and update the contents of working memory, feeding into ruminative thinking centred on negative, non-specific events. Impaired memory processes contribute to broader cognitive deficits during the acute depressive state affecting day-to-day planning and problem solving, potentially triggering subsequent maladaptive mood-congruent recall of past events. The interaction between hot and cold pathways serves to perpetuate the negative memory cycle and worsen depressive symptoms. Components of the model are informed by Beck's cognitive model of depression. 


\section{An Integrated Hot-Cold Cognitive Model}

As demonstrated above, the cold cognitive processes that underlie the negative schemas and cognitive biases proposed by Beck's cognitive model seem to largely be accounted for by two, complementary processes: (i) weakened executive control (cognitive flexibility, inhibition, working memory), which facilitates the activation and maintenance of a hot cognitive profile by means of affective interference, and (ii) further exacerbation of cold cognitive dysfunction by means of diminished cognitive resources which, in turn, likely represent internal stressors maintaining the depressive state.

The former process corroborates previous literature that cognitive biases are maintained by impoverished abilities to effectively disengage from negative material (Gotlib \& Joormann, 2010). As such, the material present in working memory tends to be of moodcongruent, negative valence due to a poor ability to inhibit the generation of subsequent negative material or flexibly shift to potentially-relevant, positive material. For the most part, literature has examined the role of weakened executive control during MDD using affective tasks in which the ability to inhibit or shift from negative material is assessed (Koster, Hoorelbeke, Onraedt, Owens, \& Derakshan, 2017). Resultantly, findings have created a narrative around various components of affective, hot cognitive processes. Our model provides a novel narrative as it includes non-affective, cold cognitive deficits in the MDD cycle to suggest that, in some circumstances, it is a general deficit that facilitates the expression of hot executive deficits towards the processing of negative material. Consistent with this, an accumulating body of literature demonstrates that cognitive deficits persist into remission (Semkovska et al., 2019), and therefore cannot merely be an epiphenomenon of mood state. Instead, these deficits are conceptualised as the means by which affective, hot deficits in cognitive domains such as attention and executive function manifest. Collectively, these factors work to initiate and maintain the depressive schemas and cognitive biases by making negative material readily accessible.

The latter process takes the form of exacerbated cold cognitive deficits, which likely act as internal stressors triggering the repeated activation of dysfunctional hot cognitions. In this way, the stressors become apparent in day-to-day functioning when deficient ability to attend, memorise, plan, and problem-solve provide a source of self-referent feedback to confirm negative schemas about the self. This sets up a self-perpetuating cycle as deficient cold cognition activates the influence of hot processing biases and a ruminative response style that collectively place large demands on cognitive resources, and in turn, deprive the allocation of resources to cold cognitions required in everyday function across work, home, and social environments. Again, cold cognitive deficits appear somewhat independent of hot schemas and biases as deficient functioning is observed even in the state of mood restoration (Evans, Iverson, Yatham, \& Lam, 2014). In the context of Beck's cognitive model, cold cognitive deficits reinforce hot cognition by providing accessible schema-congruent material (e.g. 'I am a failure', 'I am not as good as others') and likely serve as a source of maintenance for the MDD cycle. 
Our proposed model (Figure 4) postulates an interaction between cold and hot cognition in the onset and maintenance of MDD and is consistent with current neuropsychological models of MDD such as that by Roiser and Sahakian (2013). In their model, Roiser and Sahakian (2013) integrated the traditional cognitive framework with recent pharmacological findings to conceptualise MDD as the interaction between maladaptive topdown and bottom-up processes, with consideration of hot and cold components. The authors applied their findings to inform adjunctive treatment options that could address the hot/cold top-down cognition that contributes to negative expectations (e.g. CBT) or weakened executive control (e.g. transcranial magnetic stimulation), alongside the hot bottom-up cognition that accounts for negative perceptions (e.g. antidepressant medication). Our model further elaborates on this by providing a thorough, integrated account of cold cognitive abilities within the dominant cognitive framework of MDD. The proposed model lends itself to identifying novel initiatives to help interrupt the MDD cycle and best inform refined, psychological treatment options. This incorporates the consideration of hot and cold maladaptive cognitions. To date, CBT is well-established as an effective treatment for MDD by addressing the hot cognitive components that serve to maintain the depressive schemas. Several studies have shown that CBT has beneficial effects on cognitive biases of attention (e.g. Tobon, Ouimet, \& Dozois, 2011), interpretation (e.g. Williams et al., 2015), memory (e.g. McBride, Segal, Kennedy, \& Gemar, 2007), as well as maladaptive, ruminative emotion regulation strategies (for review, see Spinhoven et al., 2018). However, CBT has shown little, if any, influence on cold cognitive abilities (Groves et al., 2015; Porter et al., 2016). This possibly explains the relapse rates subsequent to treatment considering the cold cognitive aspects of the MDD cycle were not addressed. Attention training and improving control over thinking may facilitate the ability to flexibly shift attention and disengage from the negative schemas or associated biases. In support of this, cognitive remediation strategies, which explicitly target cognitive abilities shown to be deficient during MDD through repetitive and adaptive training, have shown much promise in strengthening such cold cognitive abilities (Motter, Grinberg, Lieberman, Iqnaibi, \& Sneed, 2019; Motter et al., 2016; Semkovska \& Ahern, 2017; Trapp, Engel, Hajak, Lautenbacher, \& Gallhofer, 2016). A comprehensive review by Koster et al. (2017) provides a compelling discussion on the potential means by which cognitive training affects depressive symptoms. 


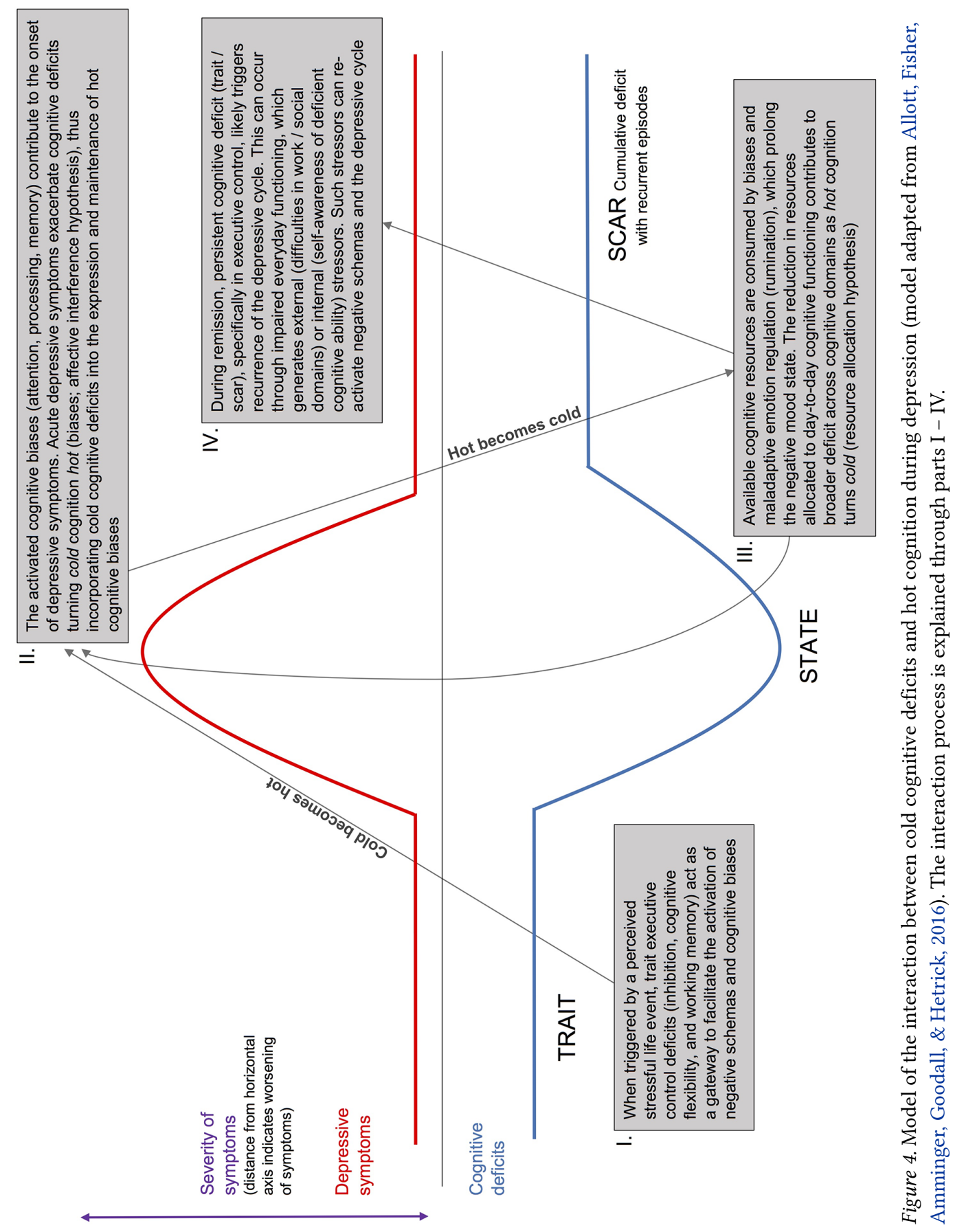


In summary, cognitive training may exert benefit by altering mediational hot cognitive factors (e.g. rumination), by targeting the underlying neurocircuitry known to be compromised during depression (for review, see Drevets, Price, \& Furey, 2008), or finally by influencing vulnerability to the activation of cognitive biases. The consideration of cognitive training as an adjunct to CBT may best complement outcomes for depressive symptoms by fully accounting for the interaction between hot and cold cognitions in the onset and maintenance of MDD. Patient preference largely favours psychological treatment over medication (McHugh et al., 2013) and this gives prominence to the need for integrated treatment options that are embedded within the cognitive theory framework.

Significant developments have been made in understanding the role of cognition in MDD, but further integrative research with the aim of refining cognitive models of MDD will be necessary to improve approaches towards prevention and treatment. No single form of cognition solely drives the depressive cycle, thus modelling the interplay between various facets of hot and cold cognition is a promising avenue to stimulate research. Researchers should endeavour to identify the relative contribution of hot and cold cognitions in interventional research to best account for the mechanisms by which symptom improvement can be achieved. This is a crucial step in transitioning from the symptom-based to the mechanism-based view of MDD (Grahek et al., 2018). In addition, consideration of hot and cold cognitive processes in longitudinal research may help to account for the directionality of effects. Cold deficits may serve as the initiating mechanism for hot cognitions, as suggested in the literature (e.g. Allott et al., 2016), but once a major depressive episode is initiated, the extent to which cold deficits are necessary for the maintenance of negative schemas and associated biases is not well understood. An important aim of our integrated model is to encourage hypothesis-driven research efforts to examine the potential of hot and cold cognitive mechanisms in novel treatment initiatives.

In conclusion, our integrated hot-cold cognitive model of MDD provides a preliminary framework to identify the central role that cold cognitive abilities play in conjunction with the more widely recognised hot cognitive processes that underpin Beck's conceptualisation of MDD onset and maintenance. The growing body of research on deficits in non-affective, cold cognitive abilities during remission confirms that cold cognition is not merely an epiphenomenon of the acute state. Here, we have proposed a re-framing of the cognitive model to account for the complex interaction between cognition and emotion during MDD. By doing so, this stimulates further opportunity to develop innovative treatment strategies that can effectively address a full functional recovery from this highly recurrent and debilitating disorder. 
Funding: This work was supported by The Irish Research Council under the Government of Ireland Postgraduate Scholarship Programme awarded to Elayne Ahern, under the supervision of Principal Investigator Maria Semkovska (GOIPG/2018/1729).

Competing Interests: The authors declare no conflicts of interest.

Acknowledgments: The authors have no support to report.

\section{References}

Ahern, E., \& Semkovska, M. (2017). Cognitive functioning in the first-episode of major depressive disorder: A systematic review and meta-analysis. Neuropsychology, 31(1), 52-72. https://doi.org/10.1037/neu0000319

Aker, M., Bø, R., Harmer, C., Stiles, T. C., \& Landrø, N. I. (2016). Inhibition and response to error in remitted major depression. Psychiatry Research, 235(1), 116-122. https://doi.org/10.1016/j.psychres.2015.11.038

Allott, K., Fisher, C. A., Amminger, G. P., Goodall, J., \& Hetrick, S. (2016). Characterizing neurocognitive impairment in young people with major depression: State, trait, or scar? Brain and Behavior, 6(10), Article e00527. https://doi.org/10.1002/brb3.527

American Psychiatric Association. (2013). Diagnostic and statistical manual of mental disorders (5th ed.). Arlington, VA, USA: American Psychiatric Publishing.

Baune, B. T., \& Renger, L. (2014). Pharmacological and non-pharmacological interventions to improve cognitive dysfunction and functional ability in clinical depression: A systematic review. Psychiatry Research, 219(1), 25-50. https://doi.org/10.1016/j.psychres.2014.05.013

Beats, B. C., Sahakian, B. J., \& Levy, R. (1996). Cognitive performance in tests sensitive to frontal lobe dysfunction in the elderly depressed. Psychological Medicine, 26(3), 591-603. https://doi.org/10.1017/S0033291700035662

Beck, A. T. (2008). The evolution of the cognitive model of depression and its neurobiological correlates. American fournal of Psychiatry, 165(8), 969-977. https://doi.org/10.1176/appi.ajp.2008.08050721

Beevers, C. G., Clasen, P. C., Enock, P. M., \& Schnyer, D. M. (2015). Attention bias modification for major depressive disorder: Effects on attention bias, resting state connectivity, and symptom change. Journal of Abnormal Psychology, 124(3), 463-475. https://doi.org/10.1037/abn0000049

Bisson, M. S., \& Sears, C. R. (2007). The effect of depressed mood on the interpretation of ambiguity, with and without negative mood induction. Cognition and Emotion, 21(3), 614-645. https://doi.org/10.1080/02699930600750715

Blackwell, S. E., Browning, M., Mathews, A., Pictet, A., Welch, J., Davies, J., . . Holmes, E. A. (2015). Positive imagery-based cognitive bias modification as a web-based treatment tool for depressed 
adults: A randomized controlled trial. Clinical Psychological Science, 3(1), 91-111. https://doi.org/10.1177/2167702614560746

Boland, R. J., \& Keller, M. B. (2009). Course and outcome of depression. In I. H. Gotlib \& C. Hammen (Eds.), Handbook of depression (2nd ed., pp. 23-43). New York, NY, USA: The Guilford Press.

Bortolato, B., Miskowiak, K. W., Kohler, C. A., Maes, M., Fernandes, B. S., Berk, M., \& Carvalho, A. F. (2016). Cognitive remission: A novel objective for the treatment of major depression? BMC Medicine, 14, Article 9. https://doi.org/10.1186/s12916-016-0560-3

Buist-Bouwman, M. A., Ormel, J., De Graaf, R., De Jonge, P., Van Sonderen, E., Alonso, J., . . . ESEMeD/MHEDEA 2000 investigators. (2008). Mediators of the association between depression and role functioning. Acta Psychiatrica Scandinavica, 118(6), 451-458.

https://doi.org/10.1111/j.1600-0447.2008.01285.x

Butler, G., \& Mathews, A. (1983). Cognitive processes in anxiety. Advances in Behaviour Research and Therapy, 5(1), 51-62. https://doi.org/10.1016/0146-6402(83)90015-2

Cheung, M. S. L., Sin, E. L. L., Lam, M., \& Lee, T. M. C. (2018). The relationship between rumination and autobiographical memory specificity in people with depression. Neuropsychiatry, 8(4), 1310-1318. https://doi.org/10.4172/Neuropsychiatry.1000460

Dalgleish, T., \& Werner-Seidler, A. (2014). Disruptions in autobiographical memory processing in depression and the emergence of memory therapeutics. Trends in Cognitive Sciences, 18(11), 596-604. https://doi.org/10.1016/j.tics.2014.06.010

De Raedt, R., \& Koster, E. H. W. (2010). Understanding vulnerability for depression from a cognitive neuroscience perspective: A reappraisal of attentional factors and a new conceptual framework. Cognitive, Affective, \& Behavioural Neuroscience, 10(1), 50-70.

https://doi.org/10.3758/CABN.10.1.50

Disner, S. G., Beevers, C. G., Haigh, E. A., \& Beck, A. T. (2011). Neural mechanisms of the cognitive model of depression. Nature Reviews. Neuroscience, 12(8), 467-477.

https://doi.org/10.1038/nrn3027

Douglas, K. M., \& Porter, R. J. (2009). Longitudinal assessment of neuropsychological function in major depression. Australian and New Zealand fournal of Psychiatry, 43(12), 1105-1117. https://doi.org/10.3109/00048670903279887

Drevets, W. C., Price, J. L., \& Furey, M. L. (2008). Brain structural and functional abnormalities in mood disorders: Implications for neurocircuitry models of depression. Brain Structure and Function, 213(1-2), 93-118. https://doi.org/10.1007/s00429-008-0189-x

Elliott, R., Sahakian, B., Herrod, J., Robbins, T., \& Paykel, E. (1997). Abnormal response to negative feedback in unipolar depression: Evidence for a diagnosis specific impairment. fournal of Neurology, Neurosurgery \& Psychiatry, 63(1), 74-82. https://doi.org/10.1136/jnnp.63.1.74

Elliott, R., Sahakian, B. J., McKay, A., Herrod, J., Robbins, T. W., \& Paykel, E. (1996).

Neuropsychological impairments in unipolar depression: The influence of perceived failure on subsequent performance. Psychological Medicine, 26(5), 975-989.

https://doi.org/10.1017/S0033291700035303 
Ellis, H. C., \& Ashbrook, P. W. (1988). Resource allocation model of the effects of depressed mood states on memory. In K. Fiedler \& J. Forgas (Eds.), Affect, cognition and social behaviour (pp. 2543). Toronto, Canada: Hogrefe.

Evans, V. C., Iverson, G. L., Yatham, L. N., \& Lam, R. W. (2014). The relationship between neurocognitive and psychosocial functioning in major depressive disorder: A systematic review. Journal of Clinical Psychiatry, 75(12), 1359-1370. https://doi.org/10.4088/JCP.13r08939

Everaert, J., Podina, I. R., \& Koster, E. H. W. (2017). A comprehensive meta-analysis of interpretation biases in depression. Clinical Psychology Review, 58(1), 33-48. https://doi.org/10.1016/j.cpr.2017.09.005

Foldi, N. S., Brickman, A. M., Schaefer, L. A., \& Knutelska, M. E. (2003). Distinct serial position profiles and neuropsychological measures differentiate late life depression from normal aging and Alzheimer's disease. Psychiatry Research, 120(1), 71-84. https://doi.org/10.1016/S0165-1781(03)00163-X

Friedman, N. P., \& Miyake, A. (2017). Unity and diversity of executive functions: Individual differences as a window on cognitive structure. Cortex, 86(1), 186-204. https://doi.org/10.1016/j.cortex.2016.04.023

Gaddy, M. A., \& Ingram, R. E. (2014). A meta-analytic review of mood-congruent implicit memory in depressed mood. Clinical Psychology Review, 34(5), 402-416. https://doi.org/10.1016/j.cpr.2014.06.001

Gamble, B., Moreau, D., Tippett, L. J., \& Addis, D. (2018, November 2). Specificity of future thinking in depression: A meta-analysis [Preprint]. https://doi.org/https://doi.org/10.31234/osf.io/y84pu

Gibbs, B. R., \& Rude, S. S. (2004). Overgeneral autobiographical memory as depression vulnerability. Cognitive Therapy and Research, 28(4), 511-526.

https://doi.org/10.1023/B:COTR.0000045561.72997.7c

Gotlib, I. H., \& Joormann, J. (2010). Cognition and depression: Current status and future directions. Annual Review of Clinical Psychology, 6, 285-312. https://doi.org/10.1146/annurev.clinpsy.121208.131305

Gotlib, I. H., Krasnoperova, E., Yue, D. N., \& Joormann, J. (2004). Attentional biases for negative interpersonal stimuli in clinical depression. fournal of Abnormal Psychology, 113(1), 127-135. https://doi.org/10.1037/0021-843X.113.1.121

Grahek, I., Everaert, J., Krebs, R. M., \& Koster, E. H. W. (2018). Cognitive control in depression: Toward clinical models informed by cognitive neuroscience. Clinical Psychological Science, 6(4), 464-480. https://doi.org/10.1177/2167702618758969

Grant, K. E., McMahon, S. D., Carter, J. S., Carleton, R. A., Adam, E. K., \& Chen, E. (2014). The influence of stressors on the development of psychopathology. In M. Lewis \& K. D. Rudolph (Eds.), Handbook of developmental psychopathology (pp. 205-223). Boston, MA, USA: Springer US.

Gronwall, D. M. A. (1977). Paced Auditory Serial Addition Task - Measure of recovery from concussion. Perceptual and Motor Skills, 44(2), 367-373. https://doi.org/10.2466/pms.1977.44.2.367 
Groves, S. J., Douglas, K. M., \& Porter, R. J. (2018). A systematic review of cognitive predictors of treatment outcome in major depression. Frontiers in Psychiatry, 9, Article 382. https://doi.org/10.3389/fpsyt.2018.00382

Groves, S. J., Porter, R. J., Jordan, J., Knight, R., Carter, J. D., McIntosh, V. V., . . Lacey, C. (2015). Changes in neuropsychological function after treatment with metacognitive therapy or cognitive behavior therapy for depression. Depression and Anxiety, 32(6), 437-444. https://doi.org/10.1002/da.22341

Hallford, D. J., Austin, D. W., Takano, K., \& Raes, F. (2018). Psychopathology and episodic future thinking: A systematic review and meta-analysis of specificity and episodic detail. Behaviour Research and Therapy, 102, 42-51. https://doi.org/10.1016/j.brat.2018.01.003

Hammar, A., Lund, A., \& Hugdahl, K. (2003). Selective impairment in effortful information processing in major depression. Journal of the International Neuropsychological Society, 9(6), 954-959. https://doi.org/10.1017/S1355617703960152

Hammen, C. (2005). Stress and depression. Annual Review of Clinical Psychology, 1, 293-319. https://doi.org/10.1146/annurev.clinpsy.1.102803.143938

Hartlage, S., Alloy, L. B., Vázquez, C., \& Dykman, B. (1993). Automatic and effortful processing in depression. Psychological Bulletin, 113(2), 247-278. https://doi.org/10.1037/0033-2909.113.2.247

Hindash, A. H. C., \& Amir, N. (2012). Negative interpretation bias in individuals with depressive symptoms. Cognitive Therapy and Research, 36(5), 502-511.

https://doi.org/10.1007/s10608-011-9397-4

Hirsch, C. R., Krahé, C., Whyte, J., Loizou, S., Bridge, L., Norton, S., \& Mathews, A. (2018). Interpretation training to target repetitive negative thinking in generalized anxiety disorder and depression. fournal of Consulting and Clinical Psychology, 86(12), 1017-1030. https://doi.org/10.1037/ccp0000310

Hitchcock, C., Golden, A.-M. J., Werner-Seidler, A., Kuyken, W., \& Dalgleish, T. (2018). The impact of affective context on autobiographical recollection in depression. Clinical Psychological Science, 6(3), 315-324. https://doi.org/10.1177/2167702617740672

Hitchcock, C., Mueller, V., Hammond, E., Rees, C., Werner-Seidler, A., \& Dalgleish, T. (2016). The effects of autobiographical memory flexibility (MemFlex) training: An uncontrolled trial in individuals in remission from depression. Fournal of Behavior Therapy and Experimental Psychiatry, 52, 92-98. https://doi.org/10.1016/j.jbtep.2016.03.012

Hoorelbeke, K., \& Koster, E. H. W. (2017). Internet-delivered cognitive control training as a preventive intervention for remitted depressed patients: Evidence from a double-blind randomized controlled trial study. Journal of Consulting and Clinical Psychology, 85(2), 135-146. https://doi.org/10.1037/ccp0000128

Jonassen, R., Harmer, C. J., Hilland, E., Maglanoc, L. A., Kraft, B., Browning, M., . . Landro, N. I. (2019). Effects of attentional bias modification on residual symptoms in depression: A randomized controlled trial. BMC Psychiatry, 19(1), Article 141.

https://doi.org/10.1186/s12888-019-2105-8 
Joormann, J. (2010). Cognitive inhibition and emotion regulation in depression. Current Directions in Psychological Science, 19(3), 161-166. https://doi.org/10.1177/0963721410370293

Joormann, J., \& Vanderlind, W. M. (2014). Emotion regulation in depression: The role of biased cognition and reduced cognitive control. Clinical Psychological Science, 2(4), 402-421. https://doi.org/10.1177/2167702614536163

Kellough, J. L., Beevers, C. G., Ellis, A. J., \& Wells, T. T. (2008). Time course of selective attention in clinically depressed young adults: An eye tracking study. Behaviour Research and Therapy, 46(11), 1238-1243. https://doi.org/10.1016/j.brat.2008.07.004

Kertz, S. J., Belden, A. C., Tillman, R., \& Luby, J. (2016). Cognitive control deficits in shifting and inhibition in preschool age children are associated with increased depression and anxiety over 7.5 years of development. Journal of Abnormal Child Psychology, 44(6), 1185-1196. https://doi.org/10.1007/s10802-015-0101-0

Knight, M. J., Aboustate, N., \& Baune, B. T. (2018). Cognitive dysfunction in major depressive disorder: Cause and effect. Current Behavioral Neuroscience Reports, 5(4), 302-309. https://doi.org/10.1007/s40473-018-0160-y

Koster, E. H. W., De Lissnyder, E., Derakshan, N., \& De Raedt, R. (2011). Understanding depressive rumination from a cognitive science perspective: The impaired disengagement hypothesis. Clinical Psychology Review, 31(1), 138-145. https://doi.org/10.1016/j.cpr.2010.08.005

Koster, E. H. W., Hoorelbeke, K., Onraedt, T., Owens, M., \& Derakshan, N. (2017). Cognitive control interventions for depression: A systematic review of findings from training studies. Clinical Psychology Review, 53, 79-92. https://doi.org/10.1016/j.cpr.2017.02.002

Lawson, C., \& MacLeod, C. (1999). Depression and the interpretation of ambiguity. Behaviour Research and Therapy, 37(5), 463-474. https://doi.org/10.1016/S0005-7967(98)00131-4

Lawson, C., MacLeod, C., \& Hammond, G. (2002). Interpretation revealed in the blink of an eye: Depressive bias in the resolution of ambiguity. Journal of Abnormal Psychology, 111(2), 321-328. https://doi.org/10.1037/0021-843X.111.2.321

Lee, R. S. C., Hermens, D. F., Redoblado-Hodge, M. A., Naismith, S. L., Porter, M. A., Kaur, M., . . . Hickie, I. B. (2013). Neuropsychological and socio-occupational functioning in young psychiatric outpatients: A longitudinal investigation. PLOS ONE, 8(3), Article e58176. https://doi.org/10.1371/journal.pone.0058176

Liu, Y., Yu, X., Yang, B., Zhang, F., Zou, W., Na, A., . . Yin, G. (2017). Rumination mediates the relationship between overgeneral autobiographical memory and depression in patients with major depressive disorder. BMC Psychiatry, 17(1), Article 103. https://doi.org/10.1186/s12888-017-1264-8

Mathews, A., \& MacLeod, C. (2005). Cognitive vulnerability to emotional disorders. Annual Review of Clinical Psychology, 1, 167-195. https://doi.org/10.1146/annurev.clinpsy.1.102803.143916

Matt, G. E., Vázquez, C., \& Campbell, W. K. (1992). Mood-congruent recall of affectively toned stimuli: A meta-analytic review. Clinical Psychology Review, 12(2), 227-255.

https://doi.org/10.1016/0272-7358(92)90116-P 
McBride, C., Segal, Z., Kennedy, S., \& Gemar, M. (2007). Changes in autobiographical memory specificity following cognitive behavior therapy and pharmacotherapy for major depression. Psychopathology, 40(3), 147-152. https://doi.org/10.1159/000100003

McHugh, R. K., Whitton, S. W., Peckham, A. D., Welge, J. A., \& Otto, M. W. (2013). Patient preference for psychological vs pharmacologic treatment of psychiatric disorders: A metaanalytic review. fournal of Clinical Psychiatry, 74(6), 595-602. https://doi.org/10.4088/JCP.12r07757

Miyake, A., Friedman, N. P., Emerson, M. J., Witzki, A. H., Howerter, A., \& Wager, T. D. (2000). The unity and diversity of executive functions and their contributions to complex "Frontal Lobe" tasks: A latent variable analysis. Cognitive Psychology, 41(1), 49-100. https://doi.org/10.1006/cogp.1999.0734

Mogg, K., \& Bradley, B. P. (2005). Attentional bias in generalized anxiety disorder versus depressive disorder. Cognitive Therapy and Research, 29(1), 29-45. https://doi.org/10.1007/s10608-005-1646-y

Mogg, K., Bradley, B. P., Williams, R., \& Mathews, A. (1993). Subliminal processing of emotional information in anxiety and depression. fournal of Abnormal Psychology, 102(2), 304-311. https://doi.org/10.1037/0021-843X.102.2.304

Mörkl, S., Blesl, C., Jahanshahi, M., Painold, A., \& Holl, A. K. (2016). Impaired probabilistic classification learning with feedback in patients with major depression. Neurobiology of Learning and Memory, 127, 48-55. https://doi.org/10.1016/j.nlm.2015.12.001

Motter, J. N., Grinberg, A., Lieberman, D. H., Iqnaibi, W. B., \& Sneed, J. R. (2019). Computerized cognitive training in young adults with depressive symptoms: Effects on mood, cognition, and everyday functioning. Fournal of Affective Disorders, 245, 28-37. https://doi.org/10.1016/j.jad.2018.10.109

Motter, J. N., Pimontel, M. A., Rindskopf, D., Devanand, D. P., Doraiswamy, P. M., \& Sneed, J. R. (2016). Computerized cognitive training and functional recovery in major depressive disorder: A meta-analysis. Journal of Affective Disorders, 189, 184-191. https://doi.org/10.1016/j.jad.2015.09.022

Murphy, F. C., Michael, A., Robbins, T. W., \& Sahakian, B. J. (2003). Neuropsychological impairment in patients with major depressive disorder: The effects of feedback on task performance. Psychological Medicine, 33(3), 455-467. https://doi.org/10.1017/S0033291702007018

Nolen-Hoeksema, S. (1991). Responses to depression and their effects on the duration of depressive episodes. Fournal of Abnormal Psychology, 100(4), 569-582. https://doi.org/10.1037/0021-843X.100.4.569

Nutt, D., Demyttenaere, K., Janka, Z., Aarre, T., Bourin, M., Canonico, P. L., . . Stahl, S. (2007). The other face of depression, reduced positive affect: The role of catecholamines in causation and cure. Journal of Psychopharmacology, 21(5), 461-471. https://doi.org/10.1177/0269881106069938

Pizzagalli, D. A., Peccoralo, L. A., Davidson, R. J., \& Cohen, J. D. (2006), Resting anterior cingulate activity and abnormal responses to errors in subjects with elevated depressive symptoms: A 128channel EEG study. Human Brain Mapping, 27, 185-201. https://doi.org/10.1002/hbm.20172 
Porter, R. J., Bourke, C., Carter, J., Douglas, K., McIntosh, V., Jordan, J., . . Frampton, C. (2016). No change in neuropsychological dysfunction or emotional processing during treatment of major depression with cognitive-behaviour therapy or schema therapy. Psychological Medicine, 46(2), 393-404. https://doi.org/10.1017/S0033291715001907

Raes, F., Hermans, D., Williams, J. M. G., Demyttenaere, K., Sabbe, B., Pieters, G., \& Eelen, P. (2005). Reduced specificity of autobiographical memory: A mediator between rumination and ineffective social problem-solving in major depression? Journal of Affective Disorders, 87(2-3), 331-335. https://doi.org/10.1016/j.jad.2005.05.004

Raes, F., Hermans, D., Williams, J. M. G., Demyttenaere, K., Sabbe, B., Pieters, G., \& Eelen, P. (2006). Is overgeneral autobiographical memory an isolated memory phenomenon in major depression? Memory, 14(5), 584-594. https://doi.org/10.1080/09658210600624614

Raes, F., Hermans, D., Williams, M. G. J., Geypen, L., \& Eelen, P. (2006). The effect of overgeneral autobiographical memory retrieval on rumination. Psychologica Belgica, 46(1-2), 131-141. https://doi.org/10.5334/pb-46-1-2-131

Rock, P. L., Roiser, J., Riedel, W., \& Blackwell, A. (2014). Cognitive impairment in depression: A systematic review and meta-analysis. Psychological Medicine, 44(10), 2029-2040. https://doi.org/10.1017/S0033291713002535

Roiser, J. P., \& Sahakian, B. J. (2013). Hot and cold cognition in depression. CNS Spectrums, 18(3), 139-149. https://doi.org/10.1017/S1092852913000072

Sanchez, A., Duque, A., Romero, N., \& Vazquez, C. (2017). Disentangling the interplay among cognitive biases: Evidence of combined effects of attention, interpretation and autobiographical memory in depression. Cognitive Therapy and Research, 41(6), 829-841.

https://doi.org/10.1007/s10608-017-9858-5

Schroder, H. S., Moran, T. P., Infantolino, Z. P., \& Moser, J. S. (2013). The relationship between depressive symptoms and error monitoring during response switching. Cognitive, Affective, \& Behavioral Neuroscience, 13(4), 790-802. https://doi.org/10.3758/s13415-013-0184-4

Semkovska, M., \& Ahern, E. (2017). Online neurocognitive remediation therapy to improve cognition in community-living individuals with a history of depression: A pilot study. Internet Interventions, 9, 7-14. https://doi.org/10.1016/j.invent.2017.04.003

Semkovska, M., Noone, M., Carton, M., \& McLoughlin, D. M. (2012). Measuring consistency of autobiographical memory recall in depression. Psychiatry Research, 197(1-2), 41-48. https://doi.org/10.1016/j.psychres.2011.12.010

Semkovska, M., Quinlivan, L., O’Grady, T., Johnson, R., Collins, A., O'Connor, J., . . Gload, T. (2019). Cognitive function following a major depressive episode: A systematic review and metaanalysis. Lancet Psychiatry. Advance online publication.

https://doi.org/10.1016/S2215-0366(19)30291-3

Shilyansky, C., Williams, L. M., Gyurak, A., Harris, A., Usherwood, T., \& Etkin, A. (2016). Effect of antidepressant treatment on cognitive impairments associated with depression: A randomised longitudinal study. Lancet Psychiatry, 3(5), 425-435.

https://doi.org/10.1016/S2215-0366(16)00012-2 
Siegle, G. J., Ingram, R. E., \& Matt, G. E. (2002). Affective interference: an explanation for negative attention biases in dysphoria? Cognitive Therapy and Research, 26(1), 73-87. https://doi.org/10.1023/A:1013893705009

Snyder, H. R. (2013). Major depressive disorder is associated with broad impairments on neuropsychological measures of executive function: A meta-analysis and review. Psychological Bulletin, 139(1), 81-132. https://doi.org/10.1037/a0028727

Snyder, H. R., \& Hankin, B. L. (2016). Spiraling out of control: Stress generation and subsequent rumination mediate the link between poorer cognitive control and internalizing psychopathology. Clinical Psychological Science: A fournal of the Association for Psychological Science, 4(6), 1047-1064. https://doi.org/10.1177/2167702616633157

Spinhoven, P., Bockting, C. L., Schene, A. H., Koeter, M. W., Wekking, E. M., \& Williams, J. M. G. (2006). Autobiographical memory in the euthymic phase of recurrent depression. fournal of Abnormal Psychology, 115(3), 590-600. https://doi.org/10.1037/0021-843X.115.3.590

Spinhoven, P., Klein, N., Kennis, M., Cramer, A. O. J., Siegle, G., Cuijpers, P., . . Bockting, C. L. (2018). The effects of cognitive-behaviour therapy for depression on repetitive negative thinking: A meta-analysis. Behaviour Research and Therapy, 106, 71-85. https://doi.org/10.1016/j.brat.2018.04.002

Steele, J. D., Kumar, P., \& Ebmeier, K. P. (2007). Blunted response to feedback information in depressive illness. Brain, 130(9), 2367-2374. https://doi.org/10.1093/brain/awm150

Tobon, J. I., Ouimet, A. J., \& Dozois, D. J. (2011). Attentional bias in anxiety disorders following cognitive behavioral treatment. fournal of Cognitive Psychotherapy, 25(2), 114-129. https://doi.org/10.1891/0889-8391.25.2.114

Trapp, W., Engel, S., Hajak, G., Lautenbacher, S., \& Gallhofer, B. (2016). Cognitive remediation for depressed inpatients: Results of a pilot randomized controlled trial. Australian \& New Zealand Journal of Psychiatry, 50(1), 46-55. https://doi.org/10.1177/0004867415622271

Van Vreeswijk, M. F., \& de Wilde, E. J. (2004). Autobiographical memory specificity, psychopathology, depressed mood and the use of the Autobiographical Memory Test: A metaanalysis. Behaviour Research and Therapy, 42(6), 731-743. https://doi.org/10.1016/S0005-7967(03)00194-3

Watkins, E., \& Teasdale, J. D. (2001). Rumination and overgeneral memory in depression: Effects of self-focus and analytic thinking. Journal of Abnormal Psychology, 110(2), 353-357. https://doi.org/10.1037/0021-843X.110.2.333

Williams, A. D., O’Moore, K., Blackwell, S. E., Smith, J., Holmes, E. A., \& Andrews, G. (2015). Positive imagery cognitive bias modification (CBM) and internet-based cognitive behavioral therapy (iCBT): A randomized controlled trial. Journal of Affective Disorders, 178, 131-141. https://doi.org/10.1016/j.jad.2015.02.026

Williams, J. M. G., Ellis, N. C., Tyers, C., Healy, H., Rose, G., \& Macleod, A. K. (1996). The specificity of autobiographical memory and imageability of the future. Memory \& Cognition, 24(1), 116-125. https://doi.org/10.3758/BF03197278 
Williams, J. M. G., Watts, F. N., MacLeod, C., \& Mathews, A. (1988). Cognitive psychology and emotional disorders. Oxford, England: John Wiley \& Sons.

Wisco, B. E., Gilbert, K. E., \& Marroquín, B. (2014). Maladaptive processing of maladaptive content: Rumination as a mechanism linking cognitive biases to depressive symptoms. Fournal of Experimental Psychopathology, 5(3), 329-350. https://doi.org/10.5127/jep.038213

World Health Organisation. (2018). Depression. Retrieved from http://www.who.int/news-room/fact-sheets/detail/depression

Yang, W., Ding, Z., Dai, T., Peng, F., \& Zhang, J. X. (2015). Attention bias modification training in individuals with depressive symptoms: A randomized controlled trial. Fournal of Behavior Therapy and Experimental Psychiatry, 49, 101-111. https://doi.org/10.1016/j.jbtep.2014.08.005

Yaroslavsky, I., Allard, E. S., \& Sanchez-Lopez, A. (2019). Can't look away: Attention control deficits predict rumination, depression symptoms and depressive affect in daily life. Journal of Affective Disorders, 245, 1061-1069. https://doi.org/10.1016/j.jad.2018.11.036

\section{EACLIPT}

Clinical Psychology in Europe (CPE) is the official journal of the European Association of Clinical Psychology and Psychological Treatment (EACLIPT).

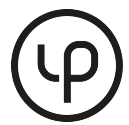

leibniz-psychology.org

PsychOpen GOLD is a publishing service by Leibniz Institute for Psychology Information (ZPID), Germany. 